\title{
MULTIPLE VENTRICULAR SEPTAL DEFECTS: HOW AND WHEN SHOULD THEY BE REPAIRED?
}

Francesco Seddio, MD

V. Mohan Reddy, MD

Doff B. McElhinney, MD

Wayne Tworetzky, MD

Norman H. Silverman, MD

Frank L. Hanley, MD
Background: Congenital heart lesions with multiple ventricular septal defects remain a surgical challenge. Traditional approaches often rely on either ventriculotomy for exposure or palliation with pulmonary artery banding. However, indications for repair versus palliation and for various approaches to surgical exposure are not clearly defined. Methods: From July 1992 to January 1998, 45 patients with multiple $(\geq 2)$ ventricular septal defects (37 with associated lesions) underwent surgery. Median age was 86 days; all but 4 patients were infants. The mean number of defects was 3.7, and almost half of the patients had more than 3 defects. Apical muscular defects were present in $62 \%$ of patients. Thirtyone patients underwent primary complete repair through a right atriotomy or trans-semilunar valve approach (group 1), 8 had palliation (group 2), and 6 underwent complete repair after prior palliation elsewhere (group 3). No patient had a ventriculotomy. Results: One early death occurred in a group 1 patient. Four patients who had had palliation $(50 \%)$ underwent early reoperation for pulmonary artery band revision because of failure to thrive or band removal after spontaneous closure of the defects. At follow-up (median 22 months), there was 1 death in a group 2 patient (palliation) and 1 other group 2 patient required cardiac transplantation. The only late reoperation was for removal of the pulmonary artery band and closure of multiple apical defects in a group 2 (palliation) patient. No patients who underwent repair have hemodynamically significant residual defects. Conclusions: In our experience, palliation of multiple ventricular septal defects is associated with greater morbidity than primary repair. Multiple defects can almost always be repaired adequately in early infancy without ventriculotomy, although "Swiss-cheese" septum may be an indication for palliation. (J Thorac Cardiovasc Surg 1999;117:134-40)
$\mathrm{M}$ ultiple ventricular septal defects (VSDs), whether isolated or associated with other cardiac abnormalities, pose a difficult management problem. Treatment options include primary surgical repair, palliative pulmonary artery banding, and transcatheter or intraoperative device closure. ${ }^{1-12}$ However, it is difficult to determine which of these techniques is most effec-

From the Divisions of Cardiothoracic Surgery a and Pediatric

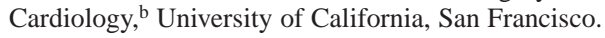

Read at the Seventy-eighth Annual Meeting of The American Association for Thoracic Surgery, Boston, Mass, May 3-6, 1998.

Received for publication May 8, 1998; revisions requested July 16, 1998; revisions received Sept 30, 1998; accepted for publication Sept 30, 1998.

Address for reprints: V. Mohan Reddy, MD, UCSF Medical Center, 505 Parnassus Ave, M593, San Francisco, CA 94143-0118.

Copyright (C) 1999 by Mosby, Inc.

$0022-5223 / 99 \$ 8.00+0 \quad \mathbf{1 2 / 6 / 9 4 8 2 0}$ tive, given the paucity of comparative reports. Over the past 6 years, our main approach has been primary repair in infancy, although a subset of patients has also undergone pulmonary artery banding. Another group of patients has undergone complete repair after primary palliation elsewhere. In the present study, we sought to examine results in infants undergoing primary closure of multiple VSDs and repair of associated lesions, in comparison with those undergoing palliation.

\section{Patients and methods}

Patients. Between July 1992 and January 1998, 45 patients with multiple $(\geq 2)$ VSDs underwent surgery. Patients were reviewed and retrospectively divided into 3 clinical groups: group 1 patients $(n=31)$ underwent primary complete repair of multiple VSDs and associated anomalies; group 2 patients $(n=8)$ underwent palliation at our institution with either pulmonary artery banding $(n=7)$ or a modified Damus-KayeStansel procedure $(n=1)$; and group 3 patients $(n=6)$ under- 
Table I. Additional cardiac abnormalities by patient group

\begin{tabular}{lcccc}
\hline $\begin{array}{l}\text { Additional } \\
\text { abnormalities }\end{array}$ & Group 1 & Group 2 & Group 3 & Total \\
\hline PDA & 3 & 1 & 0 & 4 \\
ASD & 2 & 0 & 1 & 3 \\
AVSD & 3 & 0 & 0 & 3 \\
Aortic coarctation & 3 & 2 & 0 & 5 \\
Tetralogy of Fallot & 3 & 0 & 0 & 3 \\
Pulmonary stenosis & 1 & 0 & 0 & 1 \\
Interrupted aortic arch & 1 & 0 & 1 & 2 \\
TGA/DORV & 7 & 2 & 1 & 11 \\
Other defects & 3 & 2 & 0 & 4
\end{tabular}

$P D A$, Patent ductus arteriosus; $A S D$, atrial septal defect; $A V S D$, atrioventricular septal defect; TGA, transposition of the great arteries; DORV, double-outlet right ventricle.

went complete repair after previous pulmonary artery banding performed elsewhere. Thirty-eight of the 39 patients in groups 1 and 2 were infants, with a median age of 69 days (range 6 days -2 years). The age difference between patients in groups 1 and 2 was not substantial. Four of the 6 patients in group 3 were beyond 1 year of age, with a median of 3.4 years (range 2 months-5.3 years). The median weight in groups 1 and 2 was $4.5 \mathrm{~kg}$ (range 2.1-10 kg) and in group 3, $14.5 \mathrm{~kg}$ (range $4.3-17 \mathrm{~kg}$ ).

Eight patients had isolated multiple VSDs, and 37 (82\%) had additional cardiac abnormalities (Table I). Clinical presentation varied according to associated cardiac malformations. The majority of patients in all groups had failure to thrive, congestive heart failure, cyanosis, or a combination of these symptoms, although 5 patients in group 1 and 1 patient in group 3 were free of symptoms. The diagnosis was made in all patients by means of cross-sectional and Doppler echocardiography. Diagnostic cardiac catheterization was also performed in 10 group 1 patients, 2 group 2 patients, and all group 3 patients. No patients underwent transcatheter interventional procedures.

Characterization of VSDs. Multiple VSDs were defined as more than 1 VSD. VSDs were characterized as inlet, muscular (trabecular), perimembranous, and infundibular (outlet), according to the classification of the ventricular septum published by Soto and associates. ${ }^{13}$ Muscular VSDs were characterized according to the classification proposed by Kirklin and coworkers, ${ }^{4}$ according to which posterior muscular defects are located in the inlet area (infratricuspid or inlet septal) of the muscular septum, anterior muscular defects are located anterior to the septal band of the septomarginal trabeculation, midmuscular defects are proximal to the moderator band, and apical defects are below the moderator band in the ventricular apex. We defined "Swiss-cheese" septum as multiple VSDs involving all components of the septum and/or morphologic characteristics suggestive of non-compaction. Patients with complex multiple defects involving only 1 or 2 components of the septum were not considered to have
Table II. Details of VSDs

\begin{tabular}{lcccc}
\hline Variable & Group 1 & Group 2 & Group 3 & Total \\
\hline No. of patients & 31 & 8 & 6 & 45 \\
Type of VSD* & & & & \\
$\quad$ Perimembranous & 13 & 3 & 1 & 17 \\
Inlet & 7 & 2 & 1 & 10 \\
Outlet & 1 & 2 & 0 & 3 \\
Posterior muscular & 11 & 4 & 2 & 17 \\
Anterior muscular & 11 & 4 & 3 & 18 \\
Midmuscular & 17 & 5 & 3 & 25 \\
Apical muscular & 16 & 7 & 5 & 28 \\
$\quad$ Swiss-cheese septum & 3 & 6 & 3 & 12 \\
Total No. of VSDs & 107 & 38 & 23 & 168 \\
Mean No. of VSDs & 3.5 & 4.8 & 3.8 & 3.7 \\
$\quad$ per patient & & & & \\
No. (\%) of patients & $20(64 \%)$ & $8(100 \%)$ & $5(83 \%)$ & $33(73 \%)$ \\
$\quad$ with > 2 VSDs & & & & \\
No. (\%) of patients & $11(35 \%)$ & $6(75 \%)$ & $3(50 \%)$ & $20(44 \%)$ \\
$\quad$ with > 3 VSDs & & & & \\
\hline
\end{tabular}

*Number of patients with each type of VSD.

Swiss-cheese septum. They included patients with multiple defects in only the trabecular septum, a pattern that is often referred to as Swiss-cheese VSD.

Defects in each patient were characterized on preoperative imaging and intraoperative inspection. The numbers and types of defects in each group and in the entire patient population are summarized in Table II. A large majority of patients (73\%) had more than 2 VSDs, and almost half (44\%) had more than 3 defects. The most common types of defects in all 3 patient groups were apical and midmuscular.

Operative techniques. Standard techniques of cardiopulmonary bypass with aortic and bicaval cannulation and moderate hypothermia $\left(25^{\circ} \mathrm{C}\right)$ were used in patients who underwent intracardiac repair. After institution of bypass, the aorta was crossclamped and cold crystalloid cardioplegic solution was given into the aortic root. The caval snares were then tightened and a standard right atriotomy was performed. In the patients with associated cardiac abnormalities, the technique of cardiopulmonary bypass was modified as necessary according to the additional anomalies, and additional procedures were performed as necessary. Circulatory arrest was used only in 1 patient from group 1 who underwent concurrent repair of interrupted aortic arch and closure of multiple VSDs.

The ventricular septum was examined through the tricuspid valve. A number of unique technical maneuvers typically not used for closure of perimembranous VSDs were used. One important maneuver was incision of the trabecular musculature to define complex defects in the muscular septum. The moderator band was often divided for this purpose as well. Closure of the VSDs was entirely through a right atriotomy in all patients except for 1 patient with transposition of the great arteries who had closure of an outlet defect through the pulmonary valve. In 1 case it was possible to close an anterior muscular VSD on the left ventricular aspect of the septum 
through a large perimembranous VSD that was exposed via the right atriotomy. No patient required left or right ventriculotomy. VSDs were closed either with an autologous pericardial patch (sewn in with continuous or interrupted pledgetsupported sutures, or both) or with interrupted pledgetsupported sutures. A combination of these techniques was used in 23 patients, whereas patch closure only was used in 11 and interrupted pledget-supported sutures only in 3 (all with associated defects). In 4 group 1 patients with multiple muscular VSDs, the entire trabecular septum below the moderator band was patched with a single large piece of pericardium. The patch was sewn in with a continuous 4-0 or 50 polypropylene suture, with a larger than usual needle and deeper tissue penetration to bulk up the thick trabecular muscle. Interrupted pledget-supported sutures were then used to reinforce the suture line as necessary. These same techniques were used to a lesser extent in 14 other patients, with 1 large patch used to close multiple VSDs. In 2 patients, the left side of the ventricular septum was explored through the aortic valve to make sure that all VSDs were incorporated into the closure. In these patients, a thick silk suture was passed through the VSD from the left side and pulled through from the right side to delineate the full extent of the defect. The repair was evaluated with intraoperative transesophageal echocardiography in all patients except for a $2.2-\mathrm{kg}$ infant in whom intraoperative epicardial echocardiography was used. In all patients with evidence of residual shunting by transesophageal echocardiography (except for 2 with pinpoint defects), blood samples were drawn in the operating room for superior vena caval and pulmonary arterial saturations after discontinuation of bypass and hemodynamic stabilization for the purpose of calculating the magnitude of the shunt.

In group 3 patients, who underwent closure of VSDs after primary palliation elsewhere, the pulmonary artery band was removed and the pulmonary trunk reconstructed. In 3 of these patients, patch augmentation of 1 or both branch pulmonary arteries was also necessary.

In patients undergoing palliative pulmonary artery banding (group 2), cardiopulmonary bypass was not used. Median sternotomy or thoracotomy was the surgical approach. The pulmonary trunk was dissected completely and the right and left pulmonary arteries were identified. A silicone elastomer band was passed around the pulmonary trunk, with the appropriate length determined by the formula reported by Trusler and Mustard. ${ }^{14}$ Indications for banding included avoidance of bypass in 2 infants with coarctation and multiple muscular VSDs who were admitted with profound circulatory collapse, Swiss-cheese septum with non-compaction syndrome in 2 infants, Swiss-cheese septum in 1 infant with Taussig-Bing anomaly, small right ventricle with Swiss-cheese septum in 1 infant, crisscross ventricles with multiple muscular defects in 1 infant, and multiple small muscular defects in 1 infant with transposition who was examined at 3 weeks of age.

Data analysis. Perioperative data were collected on retrospective review of patient records. Cross-sectional follow-up was conducted by contacting the referring cardiologist. Statistical analysis was performed with the use of SPSS for
Windows version 6.01 (SPSS Inc, Chicago, Ill). Dichotomous variables were compared between 2 groups of patients by means of the Fisher exact test. Continuous variables were compared between 2 groups by means of the independentsamples $t$ test. Actuarial survival analysis was performed by use of the Kaplan-Meier product limit method.

\section{Results}

Early results. One early death occurred in a 64-dayold group 1 patient who had a staged repair of coarctation and multiple VSDs during the same hospital admission. Low systemic perfusion after the coarctation repair was the rationale for the decision to close the VSDs 30 days after the coarctation repair. The patient tolerated the procedure well and was in stable condition on return to the intensive care unit. Approximately 8 hours after the operation, however, the patient had a cardiac arrest and did not respond to resuscitative efforts that included resternotomy and exploration.

Six early reoperations were required in 4 patients, all of whom were in group 2. In all 4 of these patients, the reoperation was related to the pulmonary artery band: 1 patient required band revision twice and ultimately VSD closure for failure to thrive, 2 others required revision for failure to thrive, and 1 required removal of the band after spontaneous closure of the VSDs led to decompensation 21 days after the band was placed (this patient was admitted at 3 weeks of age with transposition of the great arteries and multiple small VSDs).

No hemodynamically significant residual VSDs were detected in patients who underwent complete repair (groups 1 and 3). Small (about 1-2 mm) residual defects in the trabecular septum were seen by transesophageal Doppler echocardiography in 16 patients, 12 in group 1 and 4 in group 3 . These were primarily apical defects, although some were midmuscular or anterior. All, however, were hemodynamically insignificant, with a pulmonary/systemic blood flow ratio estimated by the Fick method at between 1.0 (no step-up in oxygen saturation from the superior vena cava to pulmonary artery despite very small defects identified by Doppler color flow echocardiography) and 1.3. It was not necessary to return to cardiopulmonary bypass to close residual defects in any patient. However, 1 patient in group 3 was returned to bypass for resection of hypertrophied infundibular muscle after intraoperative transesophageal echocardiography revealed a subvalvular gradient of $80 \mathrm{~mm} \mathrm{Hg}$, which had been obscured in the preoperative period by the presence of the pulmonary artery band. Two patients in group 1 required placement of a permanent pacemaker for complete atrioventricular block. An additional 10 patients 
had transient postoperative rhythm abnormalities, 9 in group 1 and 1 in group 3. Median hospital stay was 13.5 days in group 1 (5-32 days), 13 days in group 2 (520 days), and 9 days in group 3 (5-20 days).

Follow-up. Cross-sectional follow-up was obtained in all survivors, with a median duration of 22 months (5-55 months). There was 1 death during the follow-up period, in a group 2 patient with Taussig-Bing malformation who had methicillin-resistant Staphylococcus aureus sepsis and died 5 months after the operation. Another patient who underwent palliation required cardiac transplantation for progressive ventricular dysfunction 19 months after the operation. Actuarial survival among patients who underwent repair was $97 \%$ at 5 years.

The only late operation was for closure of the multiple VSDs and removal of the pulmonary artery band 8 months after the operation in a group 2 patient who had progressive cyanosis. At follow-up echocardiography, no patients in group 1 or 3 had significant residual VSDs, and in all but 4 patients, the small residual defects seen on intraoperative transesophageal echocardiography were no longer evident. Ventricular function was normal in all but 1 patient, who had depressed ventricular function, with an ejection fraction of $40 \%$ and septal dyskinesis. This patient had not undergone significant septal patching. Among the surviving group 2 patients who had not undergone VSD closure or transplantation, 1 patient with crisscross ventricles and 5 muscular VSDs had mildly depressed ventricular function.

\section{Discussion}

Multiple VSDs may occur as an isolated lesion or in conjunction with a variety of other congenital cardiovascular defects. Patients typically have symptoms early in infancy and often in the neonatal period if associated lesions are present. As in this report, patients with multiple VSDs may be grouped together as a single series. However, it is important to remember that management considerations can vary substantially, depending on factors such as the number, size, and location of defects, the presence and nature of associated anomalies, and the clinical status of the patient. In part because of the considerable variety seen in patients with this complex condition, the treatment of multiple VSDs remains controversial on a number of points. Primary surgical repair and palliation have been the standard approaches for many years, ${ }^{1-8}$ but transcatheter device closure provides another option. ${ }^{9,10}$ Although catheter-based techniques appear promising, they are not yet widely used for the closure of complex VSDs, and their utility may be limited at present to larger children. For the time being, at least, operative management remains the gold standard.
However, even among patients managed surgically, the optimal approach is a matter of debate.

As many surgeons have observed, it can be extremely difficult to visualize and close all of the defects in the septum from the right ventricular aspect. Before the era of Doppler color flow imaging, difficulty in preoperative identification of the multiple defects made primary repair an especially challenging endeavor. This, along with the frequent presentation in early infancy, led many to approach multiple VSDs with palliative pulmonary artery banding to provide relief from congestive heart failure. ${ }^{15-18}$

The results of our series and previous studies ${ }^{7,8}$ demonstrate that pulmonary artery banding is not indicated in the majority of patients and may in fact be detrimental both in the early postoperative period and later, at the time of complete repair. In our series, banding was accompanied by a higher incidence of early postoperative complications. Although the relatively small number of patients precluded meaningful statistical analysis, 4 of 8 patients who underwent palliation (group 2) had complications necessitating reoperation in the early postoperative period, compared with no early reoperations and 1 early death in the 37 patients who underwent primary repair. In addition to perioperative complications, patients who undergo primary pulmonary artery banding may pose management problems later as well, because they will inevitably require subsequent reoperation for removal of the band and closure of remaining defects, if applicable. As others have found, subsequent identification and closure of trabecular VSDs was much more difficult in patients in our series who underwent repair after a prior pulmonary artery band (group 3), because of hypertrophy of the ventricular septum and right ventricular trabeculae after a period of long-standing right ventricular hypertension. ${ }^{7,8}$ Right ventricular hypertrophy can also lead to subvalvular pulmonary outflow tract obstruction that may be obscured by the pulmonary artery band on preoperative evaluation, as was the case in 1 of our patients. Moreover, complications of the pulmonary artery band may occur as well, including inadequate protection of the pulmonary vascular bed and distortion of the pulmonary artery or valve, or both. For example, in a recently reported experience with pulmonary artery banding, the band was found to be inadequate at the time of follow-up in $29 \%$ of patients. ${ }^{18}$ After eventual repair in 60 patients, the mortality was substantially higher in patients with an inadequate band than those with an adequate band. In addition, pulmonary artery reconstruction is invariably necessary. Despite the many potential drawbacks of banding, there are nevertheless 
patients who are extremely ill or who have Swisscheese VSDs in whom it remains a reasonable option.

In the past, some surgeons believed that the morbidity of pulmonary artery banding compared favorably in many instances with that of surgical repair, because a transventricular approach was commonly used, leading to complications such as ventricular dysfunction, arrhythmias, or ventricular aneurysm formation in a relatively high percentage of patients. ${ }^{19}$ On the other hand, however, left ventriculotomy was and still is advocated by some as the approach of choice for closure of difficult muscular VSDs. ${ }^{2,3,5}$ In the current era, practices have evolved so that extensive ventricular incisions are generally avoided, and surgeons who do continue to use a transventricular approach tend to create a small left ventriculotomy at the apex. ${ }^{6,6 a, 8}$ Such modified methods may have fewer attendant complications than more extensive ventricular incisions, as several centers have reported. ${ }^{6,6 a, 20}$ Ultimately, only time will tell. We prefer not to take a transventricular approach, because we believe that all significant defects can be closed by means of less potentially detrimental methods.

In our experience and that of others, primary repair of multiple VSDs can be achieved with excellent results, and we recommend this approach in nearly all symptomatic patients, even neonates and premature or lowbirth-weight infants. We have found that adequate closure of all hemodynamically significant VSDs can be achieved without left or right ventriculotomy. Creative approaches will help facilitate adequate repair without a ventricular incision. For example, the left side of the interventricular septum can be visualized and often accessed through the aortic valve, mitral valve, or large perimembranous or inlet VSDs. Division of right ventricular trabeculations can aid with exposure of trabecular defects, which may be difficult to close owing to multiple sites of jet penetration through a defect as a result of the highly trabeculated right ventricular aspect of the septum. One technique to overcome this challenge is to place a single large patch over an extensive portion of the trabecular septum, which is most useful in patients with multiple muscular defects. Another useful technique, recently described by Kitagawa and colleagues, ${ }^{8}$ is to pass an oversized patch through the defect after resection of trabeculae and sew it to the left ventricular aspect of the septum by passing interrupted pledget-supported sutures through the septal muscle from the left ventricle and tying them on the right ventricular side. Kitagawa and colleagues ${ }^{8}$ also described a technique for closure of an anterior muscular defect that does not require a patch, in which interrupted pledget-supported sutures are passed through the rim of the defect, then through the right ventricular free wall, and tied on the epicardial surface of the heart to cinch the rim of the defect and the ventricular wall together. Others have reported reliable and successful closure of small muscular defects with biologic glue. ${ }^{21}$ Whichever technique is used, our experience is that all significant defects can be closed with good long-term function, except in the few patients who may have primary myocardial abnormalities.

One particularly challenging group of patients is those with so-called Swiss-cheese septum. This term may be used and interpreted in different ways, but it is often synonymous with multiple defects in the trabecular septum. However, we considered true Swiss-cheese septum to encompass morphologic features resembling ventricular non-compaction, as well as defects involving all components of the ventricular septum. According to this definition, many of the patients with multiple defects in the trabecular septum were not considered to have Swiss-cheese septum. Their VSDs were repaired by means of the techniques described with a single large patch. In patients with true Swiss-cheese septum, adequate closure of the multiple defects essentially requires ventricular septation with a patch. Their outcome may be complicated by abnormal ventricular function. One such patient in our series required heart transplantation 19 months after palliation for transposition of the great arteries with multiple VSDs. Another patient with this type of multiple muscular VSDs has depressed myocardial function at follow-up. In other series, patients have required transplantation for poor ventricular function, ${ }^{8}$ and although the specific details of these patients' anomalies were not described, they may have had some degree of ventricular non-compaction as well. This group of patients may be better served by pulmonary artery banding, with the hope that some of these defects may close as a result of ventricular myocardial hypertrophy and growth.

In conclusion, the preferred approach in almost all cases of multiple VSDs is primary closure through a right atriotomy. Excellent results can be obtained with this approach, even in neonates and low-birth-weight infants. Palliation may be appropriate in selected patients who are sufficiently ill that they cannot reasonably be expected to tolerate bypass (ie, respiratory syncytial virus pneumonia or severe systemic illness) or in cases of true Swiss-cheese septum with or without ventricular non-compaction. However, in the majority of patients palliative procedures should be avoided. They can be expected to have a higher rate of perioperative morbidity and lead to a more difficult operation at the subsequent procedure to close the remaining defects. In general, we believe that repairs performed via a ventriculotomy are suboptimal, although the ultimate 
verdict on the limited form of left ventriculotomy used in other series ${ }^{6,6 a, 8}$ is still not clear.

\section{REFERENCES}

1. Friedman WF, Mehrizi A, Pusch AL. Multiple ventricular septal defects. Circulation 1965;32:35-42.

2. Zavanella C, Matsuda H, Jara F, Subramanian S. Left ventricular approach to multiple ventricular septal defects. Ann Thorac Surg 1977;24:537-43.

3. Singh AK, de Leval MR, Stark J. Left ventriculotomy for closure of muscular ventricular septal defects: treatment of choice. Ann Surg 1977;86:577-80.

4. Kirklin JK, Castaneda AR, Keane JF, Fellows KE, Norwood WJ. Surgical management of multiple ventricular septal defects. J Thorac Cardiovasc Surg 1980;80:485-93.

5. McDaniel N, Gutgesell HP, Nolan SP, Kron IL. Repair of large muscular ventricular septal defects in infants employing left ventriculotomy. Ann Thorac Surg 1989;47:593-4.

6a. McDaniel N, Gutgesell HP, Nolan SP, Kron IL. Repair of large muscular ventricular septal defects in infants employing left ventriculotomy. Ann Thorac Surg 1997;63:288-9.

6. Wollenek G, Wyse R, Sullivan I, Elliott M, de Leval M, Stark J. Closure of muscular ventricular septal defects through a left ventriculotomy. Eur J Cardiothorac Surg 1996;10:595-8.

7. Serraf A, Lacour-Gayet F, Bruniaux J, et al. Surgical management of isolated multiple ventricular septal defects: logical approach in 130 cases. J Thorac Cardiovasc Surg 1992;103:3: 437-43.

8. Kitagawa T, Durham LA, Mosca RS, Bove EL. Techniques and results in the management of multiple ventricular septal defects. J Thorac Cardiovasc Surg. In press.

9. Bridges ND, Perry SB, Keane JF, et al. Preoperative transcatheter closure of congenital muscular ventricular septal defects. N Engl J Med 1991;324:1312-7.

10. Kumar K, Lock JE, Geva T. Apical muscular ventricular septal defects between the left ventricle and the right ventricular infundibulum: diagnostic and interventional considerations. Circulation 1997;95:1207-13.

11. Fischberger SB, Bridges ND, Keane JF, et al. Intraoperative device closure of ventricular septal defects. Circulation 1993; 88(Suppl):II205-9.

12. Murzi B, Bonanomi GL, Giusti S, et al. Surgical closure of muscular ventricular septal defects using double umbrella devices (intraoperative VSD device closure). Eur J Cardiothorac Surg 1997;12:450-4.

13. Soto B, Becker AE, Moulaert AJ, Lie JT, Anderson RH. Classification of ventricular septal defects. Br Heart J 1980;43: 332-43.

14. Trusler GA, Mustard WT. A method of banding the pulmonary artery for large isolated ventricular septal defect with and without transposition of the great arteries. Ann Thorac Surg 1972;13:351-5.

15. Stark J, Aberdeen E, Waterston DJ, Bonham-Carter BE, Tynan M. Pulmonary artery constriction (banding): a report of 146 cases. Surgery 1969;65:808-18.

16. Danilowicz D, Presti S, Colvin S, Galloway A, Langsner A, Doyle E. Results of urgent or emergency repair for symptomatic infants under one year of age with single or multiple ventricular septal defect. Am J Cardiol 1992;69:699-701.

17. Drinkwater DC, Laks H. Pulmonary artery banding. In: Baue AE, Geha AS, Hammond GL, Laks H, Naunheim KS, editors. Glenn's thoracic and cardiovascular surgery, Vol. 2. Norwalk: Appleton \& Lange; 1991. p. 955-62.
18. Pinho P, Von Oppel UO, Brink J, Hewiston J. Pulmonary artery banding: adequacy and long-term outcome. Eur J Cardiothorac Surg 1997;11:105-11.

19. Hanna B, Colan SD, Bridges ND, Mayer JE, Castaneda A. Clinical and myocardial status after left ventriculotomy for ventricular septal defect closure [abstract]. J Am Coll Cardiol 1991; 17:110A.

20. Lanford LM, Vermilion RP, Skulkin BL, Bove EL. Left ventricular function following apical left ventriculotomy for closure of muscular ventricular septal defects [abstract]. J Am Coll Cardiol 1996;27:343A.

21. Leca F, Karam J, Vouhé PR, Khoury W, et al. Surgical treatment of multiple ventricular septal defects using a biologic glue. J Thorac Cardiovasc Surg 1994;107:96-102.

\section{Discussion}

Dr Constantine Mavroudis (Chicago, Ill). Dr Seddio and his colleagues have presented an excellent series of patients with multiple VSDs who underwent surgical therapy consisting mostly of primary closure. A small group of their patients with defined indications underwent pulmonary artery banding.

This is a difficult set of patients whose therapy has varied over the years. Reports describing left ventriculotomy, right ventriculotomy, and other cavitary entry points have been described, not to mention newer therapeutic methods such as umbrella devices.

The authors have clearly demonstrated that primary closure can be accomplished even at a young age and in low-birthweight babies. To their credit, they also have offered a set of circumstances in which pulmonary artery banding is reasonable and in fact indicated.

The key to closure of multiple VSDs is accurate identification, edge detection, and proper cavitary entry. The tenets of trabecular incision and resection, interrupted suture technique, proper use of patch material, and the use of transesophageal echocardiography should allow most centers to duplicate these outstanding results. I have a few questions for the authors.

In your manuscript you made reference to the moderator band incision to locate the extent of the VSD. Anyone who does this operation recognizes that it is like trying to find a swimming pool through a forest of trees in the summer. Did you ever excise portions of the moderator band and/or associated trabeculations? If you did, did you notice significant changes in ventricular function that can be attributed to either the incision or excision of the muscle?

I am also interested in the apical VSD. Sometimes it is hard to recognize the boundaries of the defect in this kind of patient as well. Did you place sutures from the epicardial surface through the wall of the ventricle to delineate the lower end of the defect?

Last, you measured left-to-right shunts based on oxygen step-up from the right atrium and I assume the pulmonary artery. These data were supported by transesophageal color flow Doppler echocardiography. Although you did not have to resume cardiopulmonary bypass, what data points would you choose to recommence cardiopulmonary bypass and close a residual VSD?

Dr Hanley. We try not to cut the moderator band. It is usually less significant trabeculae that are farther down; I do not 
have the exact number, but we did cut the moderator band in a definable number in this series, and, yes, right bundle branch block does develop. I do not know that we were clearly able to define any reduction in right ventricular function after cutting the moderator band. Most of the time it was simply a matter of incision; occasionally we did actually cut out a small piece of muscle.

Dr Mavroudis. If you were not happy with the result, would you go back and do a pulmonary artery band at that time?

Dr Hanley. That is a judgment call with every case. If we had a pulmonary/systemic flow estimate of under 1.5:1, we would not even think of going back. If it were under $2: 1$, I would make a judgment call on the basis of whether I thought I missed something I could have or should have gotten. If the pulmonary/systemic flow ratio was substantially greater than $2: 1$, I think I would take the patient off bypass and observe how he was doing. If he was doing fine, I would not intervene. If the child had a large shunt with pulmonary hypertension, then I would probably consider inserting a band. We have not had that situation yet.

Dr Mavroudis. To treat an apical VSD, do you use sutures on the epicardial surface to bring it through?

Dr Hanley. Yes. In a few cases in this series, both for apical and for some higher anterior defects, the patch sometimes was secured transmurally, obviously making sure to avoid the coronaries.

Dr Francois Lacour-Gayet (Paris, France). We at Marie Lannelongue in Paris share the same strategy of early total repair, which we have applied to nearly 200 patients.

I have 2 questions: First, despite repeated echocardiography and even angiography, we have frequently misdiagnosed Swiss-cheese VSD. Have you met these same difficulties and on what examination do you rely?

The second question concerns low weight. In our hands, the limiting factor of closing multiple VSDs is probably not the number of VSDs to close but the viability of the residual muscular septum after closure. What is your attitude regarding very low weight?

Dr Seddio. It is difficult to detect Swiss-cheese septum by a conventional technique like transesophageal echocardiography and angiography.

Dr Hanley. It is really hard to detect Swiss-cheese septum. That is the reason we used a couple of slides to illustrate how we define Swiss-cheese septum. I do not think anybody has a really good definition. We have reserved that designation for diffuse multiple defects, essentially throughout the entire septum. Many of these patients had complex, massive, almost uncountable defects, localized to one particular area, like the apex. We are not defining that as Swiss-cheese septum. And we would merely put a large patch over that entire area, conceptually thinking about it the same way that maybe our interventional colleagues think about putting a large patch in the apex for massive multiple VSDs in the apical septum, except do it surgically, placing the patch very securely.

It is very difficult to define VSD in the pathology laboratory, and it is one step even more difficult to do it with our standard diagnostic techniques. I do not think there is an answer.
I agree with you that it is very difficult to define this condition one way or the other.

Dr Thomas L. Spray (Philadelphia, Pa). I am concerned about the definition of Swiss-cheese VSD. Multiple defects in different locations are usually addressable and are not really Swiss-cheese defects, which may be best described as "noncompaction" of the ventricular septum. If you have a true Swiss-cheese (non-compaction) defect and you use a band in that patient, then you have the problem of what to do next.

What was the overall long-term plan in the patients in your series who had initial banding with later rebanding? In multiple defects like this (in true Swiss-cheese or non-compaction defects), can you really get all of these defects closed?

If you patch the entire septum, you have to address multiple points of fixation of the patch or you end up with an aneurysmal new septum to the right of the normal septum. How do you address that issue?

I am surprised that you had so few defects noted at the completion of the operation by transesophageal echocardiography. Multiple defects are notoriously difficult to identify before the initial repair, and once you close the major defects, like the large apical or perimembranous defects, additional small or moderate-sized anterior midmuscular defects are then essentially unmasked that might require additional intervention at the same operation.

Dr Hanley. If we were on the other side of the table, I think we would be asking the same questions. All of these questions focus around how to define what is and is not Swisscheese septum. I think there really is no clear definition.

We agree with you completely that if there is a clear element of non-compaction, the true diffuse spongy ventricular septum, that clearly is defined as Swiss-cheese septum. I think it is impossible to perform septation in those patients.

Again, just to clarify the way we arbitrarily defined it in this paper: If a patient had three VSDs, an apical one, an inlet one, and an outlet one, we did not call that Swiss-cheese septum if the defects were discrete. On the other hand, many of the defects that we were able to close in this experience we think others might be calling Swiss-cheese septum because of a localized element of this spongy ventricular septum or noncompaction. However, if it were isolated to the apex, we would put one large patch over that area to take care of it.

Sixteen of the 37 patients having primary closure had residual defects. Thus I agree with you that defects can be unmasked, as well. In this particular series they were mostly quite small, with very low pulmonary/systemic flow ratios. That may be a tribute to the ability of our echocardiographic colleagues to identify either preoperatively or intraoperatively with transesophageal echocardiography all or most of the defects that would have been troublesome.

To answer Dr Lacour-Gayet's final question, yes, these are big, time-consuming operations. I would not consider it advisable to insert big, deep sutures and take up most of the septum in a 1500- or 1800-g infant. I think small size and prematurity would weigh very heavily on our minds in terms of trying to do primary closure in a patient with such complex anatomy. 